SETI Institute in Mountain View, California. "Now we also have a three-dimensional picture of how dust is distributed in the Solar System."

Most of the particles are the size of a sand grain, but a few are large enough to survive the searing heat of their passage through the atmosphere - and possibly do damage on Earth's surface. Jenniskens and his colleagues describe the discoveries in four papers accepted for publication in Icarus.

Astronomers have been documenting meteors for centuries, first by eye and more recently with radar and video-tracking systems. Meteors sprinkle Earth steadily throughout the year, but during a shower a significant number seem to originate from the same point in the sky. Skywatchers around the world have reported more than 750 possible meteor showers to the International Astronomical Union (IAU) - but only a small fraction of those have been confirmed as bona fide events.

\section{SKY SURVEILLANCE}

Jenniskens' team set up cameras at three locations in northern California to confirm or rule out these rumoured showers. The Cameras for Allsky Meteor Surveillance (CAMS) project points 60 security cameras in different directions to capture as many shooting stars as possible. Each has a relatively narrow field of view, but together they cover a broad dome of sky centred directly overhead and extending down to $30^{\circ}$ above the horizon.

"CAMS is about getting massive data sets on meteors, so you can see through all the scatter to get at those new showers," says Phil Bland, a planetary scientist at Curtin University in Perth, Australia. He helps to run a tracking network in the Australian outback that looks "The more for extremely bright we sample meteors in an effort thesky, the to recover meteorites more detailed on the ground. our picture becomes."

Since it began in 2010, CAMS has measured more than 250,000 meteors.

Of those, about three-quarters were random singletons and one-quarter came in showers. CAMS has confirmed 81 showers that were on the IAU's questionable list, and discovered 86 new ones.

Among these is one that lights up Southern Hemisphere skies in early December, and seems to radiate from the constellation Vela. It is surprisingly strong for a shower that had not been noticed before, says Jenniskens. During the March 2013 peak of a newly confirmed shower, skywatchers saw the bright flash of a rock-sized object hitting the Moon.
The CAMS team has been expanding its search by setting up smaller camera networks in the Netherlands and New Zealand. "The more we sample the sky," says Jenniskens, "the more detailed our picture becomes of what is coming in."

\section{CORRECTIONS}

The News story 'Encryption faces quantum foe' (Nature 525, 167-168; 2015) incorrectly named the location for the cryptography workshop that began on 6 September. The workshop was held at the Schloss DagstuhlLeibniz Centre for Informatics in Wadern, not the Leibniz Center for Informatics in Oktavie-Allee.

The News Feature 'Fishing for the first Americans' (Nature 525, 176-178; 2015) incorrectly credited the photo taken at Cooper's Ferry. Credit should have gone to Hayden Wilcox, not Joanne McSporran.

The News story 'Health study set to decide data policy' (Nature 525, 16-17; 2015) incorrectly stated that an $\mathrm{NIH}$ working group planned to create a blanket data-sharing policy for the Precision Medicine Initiative. It is in fact developing a policy that can accommodate participants' varying interest in seeing their own genetic information. 